\title{
Reduction in endometrial neutrophils in proximity to implanting rat blastocysts
}

\author{
P. A. W. Rogers, A. M. Macpherson and L. Beaton \\ Department of Obstetrics and Gynaecology, Monash University, Monash Medical Centre, \\ 246 Clayton Rd, Clayton, Victoria 3168, Australia
}

\begin{abstract}
Summary. An antiserum to rat neutrophils was raised and used to follow the distribution of endometrial neutrophils during the peri-implantation period. Uteri from four pregnant and four pseudopregnant rats killed at 14:00, 17:00, 20:30 and 23:00 h on day 5 of pregnancy and 09:00 h on day 6 were sectioned. Four sections from each of four implantation sites and four intersites from each rat were immunostained. There was wide variability among rats in the number of endometrial neutrophils, but a nested analysis of variance showed significantly fewer neutrophils at implantation sites than at intersites from 20:30 h onwards. This difference was primarily due to the presence of more neutrophils in intersite regions of the endometrium. The results from this study do not support a role for neutrophils in the implantation-associated increase in microvascular permeability or decidualization in rats.
\end{abstract}

Keywords: endometrium; implantation; neutrophil; rat; microvascular permeability

\section{Introduction}

One of the first signs of impending implantation in rats is a localized increase in endometrial microvascular permeability (Psychoyos, 1960; Rogers et al., 1983). The mechanism by which the embryo initiates this microvascular response is unknown.

Increased microvascular permeability and oedema are common events in implantation and inflammation (Rogers et al., 1982; Finn, 1986) and it has been hypothesized that the early components of both these complex tissue reactions may develop along similar pathways.

One cell type that often plays a major role in initiating the inflammatory response is the circulating polymorphonuclear leucocytic neutrophil (Wedmore \& Williams, 1981). Although there have been many reports of leucocyte distribution in the endometrium during the peri-implantation period (Lobel et al., 1967; Tachi \& Tachi, 1986; Sawicki et al., 1988; Noun et al., 1989), there has only been one quantitative investigation of endometrial neutrophil distribution at this time (Finn \& Pope, 1991); and from that study in mice, it was concluded that during the early stages of decidualization, shortly after the increase in microvascular permeability has commenced, there is an attraction of neutrophils from the blood into the endometrial stroma. This observation raises the possibility that neutrophils may mediate part of the endometrial response to the implanting embryo.

Since increased endometrial microvascular permeability is one of the few features that appears to be common to the implantation reaction seen in most placental mammals (Psychoyos, 1960; Deanesly, 1967; Boshier, 1970; Keys et al., 1986), it is important to know whether neutrophils play a role in species other than mice. The aim of the present study was to quantify the immunohistochemical distribution of rat endometrial neutrophils over $19 \mathrm{~h}$ starting just before any observable increase in microvascular permeability in response to implanting embryos. 


\section{Materials and Methods}

\section{Experimental animals}

Female Sprague-Dawley rats aged 3-6 months were provided with pelleted feed ad libitum and housed with the lights on from $06: 00 \mathrm{~h}$ to $20: 00 \mathrm{~h}$. Rats showing a proestrous vaginal smear were placed overnight with an intact or a vasectomized male; spermatozoa in the smear, or the presence of a vaginal plug, the following morning confirmed this as day 1 of pregnancy or pseudopregnancy, respectively. The uteri from four pregnant and four pseudopregnant rats were fixed for immunohistochemistry at each of the following times: 14:00, 17:00, 20:30 and 23:00 $\mathrm{h}$ on day 5 of pregnancy and pseudopregnancy, and $09: 00 \mathrm{~h}$ on day 6 .

\section{Experimental procedure}

Rats were anaesthetized with tribromoethanol $(30 \mathrm{mg}$ per $100 \mathrm{~g}$ body weight i.p. [stock solution: $5 \mathrm{~g} \mathrm{2,2,2-}$ tribromoethanol (Merck, Schuchardt, Germany) plus $5 \mathrm{ml}$ butan-2-ol (BDH Chemicals, Poole, Dorset, UK) working solution: $0.25 \mathrm{ml}$ stock plus $10 \mathrm{ml} 0.9 \% \mathrm{w} / \mathrm{v}$ sodium chloride]) and, $15 \mathrm{~min}$ before removal of the uterus, each rat received a $1 \mathrm{ml}$ i.v. injection of $2 \%$ Evans blue in $0.9 \%$ saline. Before removal, the uterus and its accompanying vasculature was clamped with haemostats at the ovarian and cervical ends in an attempt to maintain a normal distribution of blood within the vasculature. The clamped uterus was then excised and immersion-fixed for $24 \mathrm{~h}$ in $10 \%$ buffered formalin.

After fixation, uteri showing blue bands were cut into implantation site (areas showing biue bands) and intersite regions. All uteri without blue bands were cut into equal-sized blocks approximately $4-5 \mathrm{~mm}$ long. All tissue was processed routinely for paraffin wax embedding. Uteri from pregnant rats were serially sectioned at $5 \mu \mathrm{m}(2050$ Supercut, Reichart-Jung, Nussloch, Germany), while a short run of serial sections were taken from four different blocks for each pseudopregnant animal.

\section{Raising of antiserum to rat neutrophils}

Rat neutrophils were collected from peritoneal exudate $4 \mathrm{~h}$ after an i.p. injection of $20 \mathrm{ml} 0.2 \%$ oyster glycogen (type II, Sigma Chemical Co., St Louis, MO, USA) in sterile $0.9 \% \mathrm{NaCl}$. Animals were killed with $\mathrm{CO}_{2}$ gas and received a further injection of $40 \mathrm{ml} \mathrm{NaCl}$. The peritoneal cavity was opened, a midline incision made and exudate poured into a wide-mouthed container and centrifuged for $6 \mathrm{~min}$ at $900 \mathrm{~g}$ at room temperature. The pellet was briefly resuspended in $8 \mathrm{ml} 0.5 \%$ tri-sodium citrate to lyse red blood cells and an equal volume of $\mathrm{NaCl}$ was then added and centrifuged as before. The pellet was resuspended in $\mathrm{NaCl}$, then layered over Lympho-paque (Nyegaard \& Co., Oslo, Norway). Polymorphonuclear leucocytes (PMNs) were collected from the pellet after centrifugation for 15 min at $900 \mathrm{~g} ; \sim 30 \times 10^{6} \mathrm{PMNs}$ were obtained in this way from each rat; of these, approximately $98 \%$ were neutrophils, $2 \%$ were eosinophils and a few were macrophages as revealed by Leishman's stain.

Three New Zealand White rabbits, aged 7-13 months, were immunized with cells from $1-2$ rats in $0.5 \mathrm{ml} \mathrm{NaCl}$ mixed with an equal volume of Freund's complete adjuvant, by intramuscular injection into both hind legs. The rabbits were given booster injections about 4 weeks later with PMNs from two rats, each in Freund's incomplete adjuvant, and subsequently at intervals of 8-12 weeks. Animals were bled $(10-30 \mathrm{ml})$ from a marginal ear vein every 1-2 weeks to test for antibody titre, commencing 1 week after the first boost. Antineutrophil serum for this study was derived from blood obtained 2-5 weeks after the second boost. Collected blood was allowed to coagulate and the resulting serum was heat inactivated at $56^{\circ} \mathrm{C}$ for $30-40 \mathrm{~min}$. Serum from the three rabbits was pooled after adsorption and antibody titre testing.

\section{Adsorption of anti-neutrophil serum}

Haemagglutinins were removed from the serum by adsorption with rat red blood cells from blood obtained by cardiac puncture and collected into EDTA tubes. After centrifugation of the blood, the plasma layer was discarded, the buffy coat layer was transferred to Dulbecco's phosphate-buffered saline (PBS), and $1 \mathrm{ml}$ packed red blood cells was added to $5 \mathrm{ml}$ antineutrophil serum; 2-3 adsorptions for 50-60 min with fresh erythrocytes at room temperature were carried out. Rat antilymphocyte antibodies were removed by adsorption $(2 \times 1 \mathrm{~h})$ with lymphocytes from the buffy coat layer that had been layered over Lympho-paque, spun, and collected from the interface. Lymphocytes from approximately $1.5 \mathrm{ml}$ blood were used to adsorb $1 \mathrm{ml}$ antineutrophil serum.

An aliquot of adsorbed antineutrophil serum was also adsorbed against lysed peritoneal exudate PMNs to determine whether neutrophil-specific antibodies could be removed as determined by the abolition of immunohistochemical staining. PMNs from one rat were washed and resuspended in $1 \mathrm{ml}$ distilled water, left to stand for $10 \mathrm{~min}$ then sonicated for $30 \mathrm{~s}$. Antineutrophil serum $(60 \mu \mathrm{l})$ was added to the resulting solution and left at $37^{\circ} \mathrm{C}$ for $2 \mathrm{~h}$, then spun at approximately 12000 r.p.m. (Eppendorf centrifuge $5414 \mathrm{~S}$ ) for $5 \mathrm{~min}$. The supernatant was collected and stored frozen until used. 


\section{Titration of antineutrophil serum}

In vitro activity of serum adsorbed against erythrocytes and lymphocytes was assessed by leucoagglutination titration (Simpson \& Ross, 1971). Titres against PMNs were determined in serial twofold dilutions of antineutrophil serum from 1/20 to $1 / 1280$ in $250 \mu \mathrm{l}$ PBS $\left(0.01 \mathrm{~mol} \mathrm{l}^{-1}\right)$, $\mathrm{pH} 7.4$ set up in duplicate in 24-well culture plates. Approximately $2 \times 10^{5}$ PMNs washed in $\mathrm{NaCl}$ after collection from peritoneal exudate were added in $10 \mu \mathrm{l}$ aliquots to each well. Plates were shaken, then left to stand to incubate at room temperature for $1-2 \mathrm{~h}$. Agglutination was assessed by examination under an inverted microscope at $\times 100$. The end point was taken as the greatest dilution of antineutrophil serum containing distinct clumps of cells, 10 or more cells per clump, compared with normal rabbit serum controls, which contained mainly single cells.

Adsorbed antineutrophil serum was also examined for cytotoxic antibodies to neutrophils by adding $2 \times 10^{5}$ exudate PMNs in $250 \mu \mathrm{l}$ Dulbecco's PBS with various dilutions of antineutrophit serum, with $10 \%$ heat-inactivated rat serum and $10 \%$ fresh rabbit serum as a complement source. After incubation of the 24 -well plates for $30 \mathrm{~min}$ at $37^{\circ} \mathrm{C}$, a drop from each well was mixed with a drop of $0.2 \%$ trypan blue and examined under a haemocytometer for cellular uptake of the dye.

\section{Immunohistochemistry}

For this study, implantation sites were defined by the presence of a blastocyst in the lumen of the uterus. Intersites were defined as a non-blue band area (in uteri in which blue bands were present), or a section of the uterus at least $1 \mathrm{~mm}$ lengthways from the nearest blastocyst in uteri taken from pregnant animals before the Evans Blue response had occurred. Uteri in which the lumen was open and the blastocyst was no longer clearly attached to the epithelium were rejected.

In uteri from pregnant rats, every tenth section was stained by routine haematoxylin and eosin to confirm the presence or absence of a blastocyst. A minimum of four blastocysts was located for study in each uterus. Four serial sections from each implantation site were then selected for immunohistochemical staining with antineutrophil serum. Sequences of four serial sections were also selected for immunohistochemical staining from four intersite regions from each pregnant rat and from four randomly selected regions from the uterus of each pseudopregnant rat.

For immunohistochemical staining, sections were dewaxed, rehydrated, washed in PBS for $10 \mathrm{~min}$, treated for $10 \mathrm{~min}$ in peroxidase blocking solution (one part $30 \%$ hydrogen peroxidase to nine parts absolute methanol), washed three times in PBS and then incubated with antineutrophil serum diluted 1:500 in PBS for $45 \mathrm{~min}$ at $37^{\circ} \mathrm{C}$. The primary antibody solution contained $10 \%$ non-immune fetal calf serum as a serum blocking solution. Primary antibody binding to neutrophils was visualized with a routine commercial kit (Zymed, CA, USA) using a biotinylated second antibody (incubation for $10 \mathrm{~min}$ at room temperature) followed by a streptavidin-peroxidase conjugate ( $5 \mathrm{~min}$ incubation at room temperature) and a substrate chromogen mixture (15 min incubation at room temperature with aminoethyl carbazole). Sections were not counterstained, to make neutrophil counting easier, and were mounted with aqueous mounting solution and a cover slip. Negative controls were performed by substituting antineutrophil serum with nonimmune normal rabbit serum. Neutrophils were counted in endometrial tissue only and did not include the myometrium.

\section{Statistical analysis.}

It was assumed that the data were normally distributed with a constant variance and that the observations were independent. A graphical assessment of these assumptions showed that they appeared to be valid. A transformation of the data showed no change to these conclusions, hence we undertook statistical analysis on the untransformed data. The data for implantation sites and intersites from pregnant rats were analysed by a nested analysis of variance, with the neutrophil counts for the four sections nested within each site, and the eight sites (four implantation, four intersite) nested within each rat. Differences between times were tested against inter-rat variation, and differences between implantation sites and intersites were tested against inter-site variation within each rat. Data from pseudopregnant rats could not be included in this type of nested analysis.

\section{Results}

\section{Characterization of antineutrophil serum in vitro}

Serum adsorbed against erythrocytes and lymphocytes had a leucoagglutination titre against rat neutrophils of approximately $1 / 160-1 / 320$ and possessed cytotoxic antibodies against neutrophils in the presence of complement. Antineutrophil cytotoxicity occurred at dilutions of serum from $1 / 640$ to $1 / 1280$, virtually $100 \%$ of neutrophils staining with trypan blue at these levels. Antineutrophil serum tested by immunohistochemistry against various purified populations of formalin-fixed, paraffin-embedded leucocytes showed $>95 \%$ staining of neutrophils and $<5 \%$ 
staining of lymphocytes or eosinophils. Serum adsorbed against PMNs and tested by immunohistochemistry showed approximately $90 \%$ reduced staining.

\section{Appearance of Evans blue bands}

No blue bands were visible in uteri taken from rats at 14:00 h on day 5 of pregnancy, but by 17:00 $\mathrm{h}$ all four rats had uteri with at least some faint blue bands. From 20:30 h onwards, all uteri showed distinct, evenly spaced blue bands. Loss of the zona pellucida, as assessed from haematoxylin and eosin sections, was more varied from rat to rat at each sample time, but tended to be similar for all the blastocysts within each individual uterus. All blastocysts in two of the rats at 14:00 h on day 5 of pregnancy were still zona intact, in the other two rats killed at this time, there was evidence of the remnants of some zona material on about $50 \%$ of the blastocysts. At 17:00 h, all the blastocysts in one rat that showed faint blue bands were still surrounded by a zona. In two of the other rats at this time, there was no evidence of zonae and in the fourth rat there was evidence of zona material around $50 \%$ of the blastocysts. From $20: 30 \mathrm{~h}$ on day 5 of pregnancy, all blastocysts had hatched.

\section{Immunohistochemistry}

The large inter-rat variability in number of endometrial neutrophils (Table 1, Fig. 1) found in tiais study necessitated the use of a nested analysis of variance to allow meaningful statistical interpretation of the data.

Table 1. Average number of immunoreactive neutrophilis $( \pm \mathrm{SD})$ per $5 \mu \mathrm{m}$ section of the endometrium for five groups ( $n=4$ per group) of pregnant and pseudopregnant rats sampled at different times during the peri-implantation period

\begin{tabular}{lcccrr}
\hline $\begin{array}{l}\text { Time of sampling } \\
\text { (h) }\end{array}$ & $\begin{array}{c}14: 00 \\
\text { Day } 5\end{array}$ & \multicolumn{1}{c}{$\begin{array}{c}17: 00 \\
\text { Day } 5\end{array}$} & \multicolumn{1}{c}{$\begin{array}{c}20: 30 \\
\text { Day } 5\end{array}$} & \multicolumn{1}{c}{$\begin{array}{c}23: 00 \\
\text { Day } 5\end{array}$} & \multicolumn{1}{c}{$\begin{array}{c}09: 00 \\
\text { Day } 6\end{array}$} \\
\hline Implantation & $6 \cdot 7 \pm 2 \cdot 1$ & $5 \cdot 6 \pm 2 \cdot 9$ & $13 \cdot 7 \pm 6 \cdot 0$ & $7 \cdot 6 \pm 3 \cdot 1$ & $1 \cdot 3 \pm 1 \cdot 8$ \\
$\quad$ site & $8 \cdot 4 \pm 2 \cdot 3$ & $5 \cdot 5 \pm 4 \cdot 2$ & $5 \cdot 5 \pm 2 \cdot 7$ & $5 \cdot 8 \pm 3 \cdot 0$ & $1 \cdot 4 \pm 1 \cdot 7$ \\
& $5 \cdot 7 \pm 2 \cdot 4$ & $2 \cdot 8 \pm 1 \cdot 8$ & $7 \cdot 6 \pm 3 \cdot 0$ & $3 \cdot 9 \pm 2 \cdot 6$ & $14 \cdot 4 \pm 4 \cdot 0$ \\
& $2 \cdot 2 \pm 1 \cdot 9$ & $6 \cdot 8 \pm 4 \cdot 0$ & $6 \cdot 5 \pm 2 \cdot 7$ & $11 \cdot 9 \pm 3 \cdot 6$ & $4 \cdot 3 \pm 2 \cdot 2$ \\
Intersite & $7 \cdot 2 \pm 3 \cdot 7$ & $7 \cdot 0 \pm 4 \cdot 3$ & $29 \cdot 8 \pm 8 \cdot 3$ & $9 \cdot 8 \pm 6 \cdot 0$ & $13 \cdot 8 \pm 15 \cdot 0$ \\
& $4 \cdot 4 \pm 2 \cdot 6$ & $5 \cdot 9 \pm 3 \cdot 2$ & $7 \cdot 1 \pm 2 \cdot 5$ & $9 \cdot 2 \pm 5 \cdot 3$ & $2 \cdot 4 \pm 2 \cdot 2$ \\
& $4 \cdot 0 \pm 2 \cdot 4$ & $5 \cdot 6 \pm 2 \cdot 5$ & $8 \cdot 8 \pm 4 \cdot 0$ & $7 \cdot 1 \pm 3 \cdot 2$ & $26 \cdot 1 \pm 7 \cdot 6$ \\
& $1 \cdot 3 \pm 1 \cdot 4$ & $8 \cdot 8 \pm 3 \cdot 2$ & $6 \cdot 6 \pm 3 \cdot 1$ & $17 \cdot 4 \pm 5 \cdot 7$ & $6 \cdot 4 \pm 2 \cdot 3$ \\
Pseudopregnant & $3 \cdot 2 \pm 1 \cdot 6$ & $33 \cdot 8 \pm 15 \cdot 7$ & $3 \cdot 5 \pm 3 \cdot 4$ & $18 \cdot 0 \pm 3 \cdot 7$ & $17 \cdot 9 \pm 16 \cdot 6$ \\
& $9 \cdot 7 \pm 4 \cdot 0$ & $4 \cdot 1 \pm 2 \cdot 4$ & $6 \cdot 8 \pm 4 \cdot 6$ & $3 \cdot 3 \pm 1 \cdot 4$ & $29 \cdot 0 \pm 13 \cdot 8$ \\
& $42 \cdot 8 \pm 4 \cdot 8$ & $23 \cdot 1 \pm 9 \cdot 1$ & $19 \cdot 9 \pm 7 \cdot 0$ & $13 \cdot 1 \pm 5 \cdot 4$ & $29 \cdot 6 \pm 8 \cdot 5$ \\
& $2 \cdot 4 \pm 1 \cdot 3$ & $3 \cdot 3 \pm 1 \cdot 7$ & $8 \cdot 9 \pm 3 \cdot 3$ & $14 \cdot 6 \pm 4 \cdot 4$ & $4 \cdot 3 \pm 1 \cdot 8$ \\
\hline
\end{tabular}

Each data point represents counts from 16 different sections from the same rat. Results from each of the 20 pregnant rats, for implantation site and intersite neutrophils, have been placed in the same respective positions under the appropriate headings (see also Fig. 1).

The nested analysis of variance gave a significant difference in the frequency of neutrophils between implantation sites and intersites $(P<0.001)$ and showed a significant interaction between time and sample site for neutrophil frequency in pregnant rats $(P<0.01)$. There was no significant difference in number of neutrophils between implantation sites and intersites at 14:00 and 17:00 $\mathrm{h}$ on day 5 of pregnancy, but at each sample time from $20: 30 \mathrm{~h}$ there were significantly more neutrophils at intersites than at the corresponding implantation sites. There was a significant $(P<0.05)$ increase in neutrophils at intersites between 14:00 and 20:30 h, indicating that the differences between implantation sites and intersites from $20: 30 \mathrm{~h}$ were primarily due to an increase in neutrophils at intersites rather than to a decrease in neutrophils at implantation sites. 


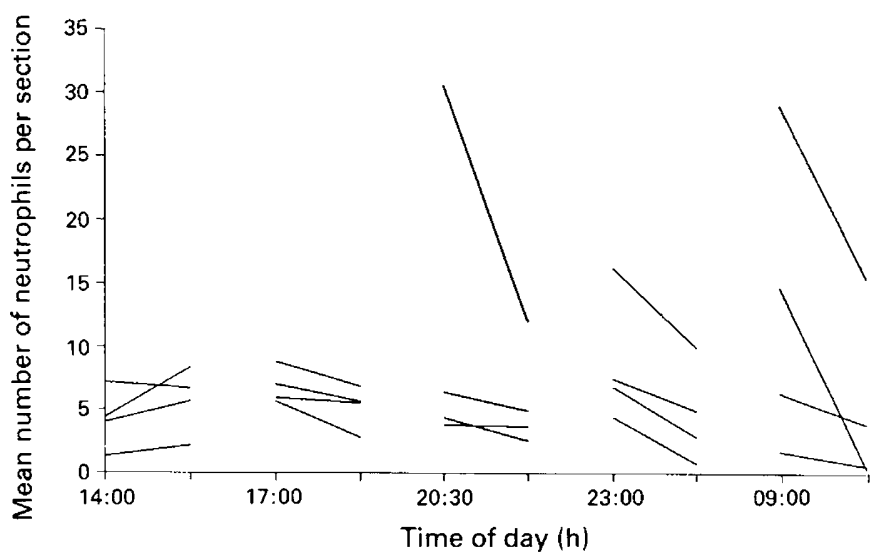

Fig. 1. Change in numbers of neutrophils between intersite and implantation site regions of the endometrium in 20 individual pregnant rats sampled at different times over the periimplantation period. Each line represents one rat. The start of each line is the average number of immunostained neutrophils per $5 \mu \mathrm{m}$ section for 16 sections from four different intersite regions, and the end of each line is the average for 16 sections from four implantation sites in the same rat.

\section{Discussion}

The results from this study demonstrate that there is no increase in endometrial neutrophils, as demonstrated by immunohistochemistry, around the implanting rat blastocyst at the time of the localized increase in microvascular permeability. Rather, as implantation develops, the numbers of neutrophils at the implantation site remain relatively constant, despite an increase in numbers in intersite regions of the endometrium. The results of the present study support those published by Noun et al. (1989), who showed by immunohistochemical methods using antibodies to T cells and granulocyte-macrophage surface markers that, at the early implantation period (days 5 to 7 ) there were no labelled cells around the conceptus. This latter study sampled only the endometrium at intervals of $24 \mathrm{~h}$; hence conclusions cannot be drawn about the specific distribution of neutrophils during the development of the implantation reaction.

The situation in rats appears to differ from that reported in mice (Finn \& Pope, 1991), where there is a significant influx of endometrial neutrophils at a similar stage of implantation. This difference between the two species, with similar modes of implantation, is striking enough to support the concept that different local mechanisms are acting during early implantation in rats and mice.

The results from this study also demonstrate that, in some individual rats, the implanting blastocyst can induce the endometrial Evans blue response before hatching from its zona pellucida. A similar observation has been reported in mice (McLaren, 1969), although in both studies a small hole or slit in the zona may have been missed because of the histological methods used to assess its integrity. Despite this, our observation supports the concept that the localized increase in endometrial microvascular permeability around the implanting blastocyst can be produced by, or at least initiated by, a substance released from the embryo that diffuses through the zona, rather than by a mechanism mediated by contact between the embryo and epithelial cells.

A number of studies have demonstrated characteristic distribution patterns for rat uterine leucocytes during the oestrous cycle and early pregnancy (Lobel et al., 1967; Tachi \& Tachi, 1986; Sawicki et al., 1988; Noun et al., 1989). Taken together, this work clearly shows that endometrial leucocyte populations are closely controlled and that these control mechanisms are under the influence of oestrogen and progesterone. The present data indicate that the embryo may also, but 
at a more local level, influence leucocyte distribution. However, this regulation of endometrial leucocyte populations is superimposed against a background of individual variability in total number of leucocytes. This variability was noted by Finn \& Pope (1991) in mice and was seen in the present study, particularly in pseudopregnant animals. We have no explanation for this large, individual variability in numbers of endometrial leucocytes, although it is important to take it into account both when designing experiments to look at this issue and when interpreting the results from such studies.

Despite a statistically significant reduction in implantation site neutrophils compared with intersite regions, the results of the present study tend to disprove a role for neutrophils in the microvascular, or other local, endometrial responses to the implanting embryo. The most obvious reason for this statement is that, on the basis of results from the current study, endometrial microvascular permeability clearly starts to increase before there is any significant change in neutrophil distribution. It is possible that neutrophil recruitment from the blood vessels into the endometrial stroma is reduced during early implantation, but that the pre-existing endometrial population remains, despite the short tissue half-life of this cell type, to facilitate the characteristic increase in microvascular permeability. Alternatively, there may be a high turnover of endometrial neutrophils at this time, despite a low immunohistochemical count at any given time. It is also possible that the antigenicity of the endometrial neutrophils is reduced at implantation sites, although the use of polyclonal antiserum in this study, rather than a monoclonal antibody, would tend to oppose this suggestion.

This study was supported by NH\&MRC grant No. 910501. We thank P. I. McCloud and P. Y. Young from the Monash University Statistical Consulting Service for help with the statistical analysis of results.

\section{References}

Boshier, D.P. (1970) The pontamine blue reaction in pregnant sheep uteri. Journal of Reproduction and Fertility 22, 595-596.

Deanesly, R. (1967) The role of the fertilized egg: reactions in the guinea-pig uterus at ovo-implantation and after thread traumatization. Journal of Reproduction and Fertility 14, 243-248.

Finn, C.A. (1986) Implantation, menstruation and inflammation. Biological Review's 61, 313-328.

Finn, C.A. \& Pope, M.D. (1991) Infiltration of neutrophil polymorphonuclear leucocytes into the endometrial stroma at the time of implantation of ova and the initiation of the oil decidual cell reaction in mice. Journal of Reproduction and Fertility 91, 365-369.

Keys, J.L., King, G.J. \& Kennedy, T.G. (1986) Increased uterine vascular permeability at the time of embryonic attachment in the pig. Biology of Reproduction 34, 405-411.

Lobel, B.L., Levy, E. \& Shelesnyak, M.C. (1967) Studies on the mechanism of nidation, XXXIV Part 3, Implantation. Acta Endocrinologica Supplementum 123, 77-109.

McLaren, A. (1969) Can mouse blastocysts stimulate a uterine response before losing the zona pellucida? Journal of Reproduction and Fertility 19, 199-201.

Noun, A., Acker, G.M., Chaouat, G., Antoine, J.C. \& Garabedian, M. (1989) Cells bearing granulocytemacrophage and $T$ lymphocyte antigens in the rat uterus before and during ovum implantation. Clinical and Experimental Immunology 78, 494-498.
Psychoyos, A. (1960) La réaction déciduale est précédée de modifications précoces de la perméabilité capillaire de l'utérus. Comptes rendu des Séances de la Société de Biologie 154, 1384-1387.

Rogers, P.A.W., Murphy, C.R. \& Gannon, B.J. (1982) Changes in the spatial organization of the uterine vasculature during implantation in the rat. Journal of Reproduction and Fertility 65, 211-214.

Rogers, P.A.W., Murphy, C.R., Rogers, A.W. \& Gannon, B. (1983) Capillary patency and permeability in the endometrium surrounding the implanting rat blastocyst. International Journal of Microcirculation: Clinical and Experimental 2, 241-249.

Sawicki, W., Choroszewska, A., Bem, W. \& Strojny, P. (1988) Lymphocyte number and distribution in the rat uterine epithelium during estrous cycle and early pregnancy. Cell and Tissue Research 253, 241-244.

Simpson, D.M. \& Ross, R.R. (1971) Effects of heterologous antineutrophil serum in guinea pigs. American Journal of Pathology 65, 79-102.

Tachi, C. \& Tachi, S. (1986) Macrophages and implantation. Annals of New York Academy of Sciences 476, 158-182.

Wedmore, C.V. \& Williams, T.J. (1981) Control of vascular permeability by polymorphonuclear leucocytes in inflammation. Nature 289, 646-650. 